\title{
Uveitis, fundamentals and clinical practice 4th edition by Robert B. Nussenblatt and Scott M. Whitcup. 480 pp. ISBN: 978-1-4377-0667-3 Elsevier/Mosby
}

\author{
Shree K. Kurup
}

Received: 7 April 2011 / Accepted: 11 April 2011 /Published online: 14 January 2012

(C) Springer-Verlag 2012

The 4th edition represents an updated version of a popular textbook (book) in ocular immunology detailing the principles and practice of therapeutics of uveitis. The authors have extensive aggregated experience in the management of severe uveitic disease. It is significant that the chapters are distributed in authorship between the two authors Nussenblatt and Whitcup, who are both wellknown in ophthalmology and immunology circles. This book has an international audience, and is designed for a wide spectrum audience. Although by convention a book is dated at conception, some of the chapters have been made sufficiently current (including online access) to avoid this fate, and thus the book provides value to the uveitis researcher. It is of interest that the authors have attempted not only to teach, but also to pass on the quality of critical thinking that makes them leaders (e.g,. lack of vitreal cells in ocular histoplasmosis)

There is a natural flow and rhythm in the order of the chapters. The words are carefully chosen, and the artwork, with accompanying illustrative and descriptive tables, is well-designed. The images are crisp, and captioned appropriately to ensure a selective quick read in the clinic, abandoning the isolated positioning of images as appendices which was evident in earlier editions. The introductory chapters are well-designed, and updated from the last edition. The use of color has enhanced the quality of the figures and made complex diagrams more reader-friendly. The chapter dealing with fundamental principles in immunology has been laid down quite succinctly and serves as a

\footnotetext{
S. K. Kurup $(\bowtie)$

Wake Forest University Eye Center,

Winston Salem, NC 27157, USA

e-mail: skurup@wfubmc.edu
}

series of valuable basic immunology mini-refresher courses. The smooth transition to the 'meat' of the clinical sciences, detailing short concise descriptions of major entities, attests to the intelligent editing. There are various methods of classifying uveitis diseases, and the book uses both anatomical and disease entity classification (i.e., anterior, posterior intermediate as well as sarcoidosis, MS). As a result, for the beginner it may be confusing to read a small paragraph on peripheral retinal abnormalities in MS. However, this criticism must be tempered with the comprehensive range of material and international threats that had to be covered, which may explain the disproportionate breadth of discussion of diseases such as yaws. There are very few editing flaws (use of trade name for bevacizumab in Fig 15-5, lumbar "function" in table on pg. 61)

Although the defined physical boundaries of a treatise limit the extent of discussion, it may be relevant in understanding disease entities. Some common disease conditions have been given less credit. Neuroretinitis is quite commonly encountered, and it would be reasonable to expect a more structured chapter on this malady. Perhaps this book would be rendered even more useful if there were a chapter dedicated to imaging in uveitic patients. In this day of high technology permeating clinical sciences, it would be gratifying to be able to answer pertinent questions in imaging analysis.

Overall, this is an indispensible volume for any reader interested in the science and therapeutics involved in ocular immunology. Over time, succeeding editions have immeasurably improved the utility and intrinsic worth of this book, providing guidance to senior practitioners and students of this complex specialty. This book is likely to be even more popular, and is much more interesting than the competition. 